\title{
Overexpression of Rab27B is correlated with distant metastasis and poor prognosis in ovarian cancer
}

\author{
PING REN $^{1 *}$, XIAO-QING YANG ${ }^{1 *}$, XIAO-LU ZHAI ${ }^{2}$, YU-QUAN ZHANG ${ }^{1}$ and JIAN-FEI HUANG ${ }^{2}$ \\ Departments of ${ }^{1}$ Obstetrics and Gynecology, and ${ }^{2}$ Pathology, \\ Affiliated Hospital of Nantong University, Nantong, Jiangsu 226001, P.R. China
}

Received November 30, 2014; Accepted December 16, 2015

DOI: $10.3892 / \mathrm{ol} .2016 .4801$

\begin{abstract}
The secretory small guanosine-5'-triphosphate-binding enzyme, Rab27B, has been identified to be an oncogene that is involved in the progression of certain tumors. The current study was designed to evaluate the expression pattern of Rab27B in ovarian cancer (OC), borderline tumors and benign ovarian adenoid tumors, as well as its association with survival prognosis and clinical parameters. The expression of Rab27B protein was examined by immunohistochemistry in 204 patients who had undergone ovarian resection without preoperative systemic chemotherapy at the Surgical Department of the Affiliated Hospital of Nantong University (Nantong, China), including 57 benign ovarian adenoid tumors, 44 borderline tumors and 103 malignant tumors. Rab27B expression and clinicopathological features were analyzed with the $\chi^{2}$ test. Patient survival rate was analyzed with the Kaplan-Meier method. Univariate and multivariate analysis of the prognostic factors was performed using the Cox regression model. Increased expression of Rab27B was positively correlated with histological type $(\mathrm{P}=0.012)$, level of differentiation $(\mathrm{P}=0.015)$, lymph node metastasis $(\mathrm{P}=0.024)$, distant metastasis $(\mathrm{P}<0.001)$ and International Federation of Gynecology and Obstetrics stage $(\mathrm{P}=0.001)$. Survival analysis revealed an association between Rab27B-positivity and poor overall survival rate. Multivariate analysis indicated that $\mathrm{Rab} 27 \mathrm{~B}$ $(\mathrm{P}<0.031)$ and distant metastases $(\mathrm{P}=0.031)$ were independent prognostic factors for $\mathrm{OC}$ patients' survival. The results of the
\end{abstract}

Correspondence to: Dr Yu-Quan Zhang, Department of Obstetrics and Gynecology, Affiliated Hospital of Nantong University, 20 Xisi Road, Nantong, Jiangsu 226001, P.R. China

E-mail: jsnt_zhangyuquan@163.com; 347943900@qq.com

Dr Jian-Fei Huang, Department of Pathology, Affiliated Hospital of Nantong University, 20 Xisi Road, Nantong, Jiangsu 226001, P.R. China

E-mail: ntyydoctor@126.com

*Contributed equally

Key words: Rab27B, ovarian cancer, immunohistochemistry, prognosis, metastasis present study supported the hypothesis that Rab27B may be a valuable prognostic indicator in patients with OC.

\section{Introduction}

Gynecological cancers are a frequently occurring group of malignancies in women, which account for $\sim 18 \%$ of all female cancers globally (1). Ovarian cancer (OC) is the most lethal common gynecological malignancy, with $>204,000$ new cases and 125,000 associated mortalities annually. Furthermore, OC accounts for 4 and $4.2 \%$ of the total cancer cases and cancer-associated mortalities in women worldwide, respectively (2). Although OC is the primary cause of cancer-associated mortality among all gynecological malignancies (3), its etiology remains to be elucidated. Matsuda et al (4) demonstrated that the 5-year relative survival rates of the age group of 15-44 years were increased compared with other age groups; however, the factors that affect five-year survival rate remain to be elucidated.

Rat sarcoma (Ras)-associated binding (Rab) protein is a member of the Ras family of small guanosine-5'-triphosphate (GTP)-binding enzymes (GTPases; 20-29 kDa). The human Rab family, which constitutes the largest family of small GTPases, includes $>60$ members that have a significant role in endocytosis, exocytosis and vesicle-trafficking (5). Rab GTPases function as molecular switches that cycle between active GTP-bound and inactive guanosine diphosphate-bound conformational states $(6,7)$. Rab proteins have a role in the transport of cargo and vesicle formation, enabling the exchange of macromolecules and proteins between various compartments and the endomembrane system (8). Rab proteins function as mediators of the complex events involved in the accurate docking and fusion of transport vesicles with their targets (9). Numerous studies have demonstrated that vesicle trafficking and exocytosis have significant roles in tumorigenesis (10). Rab27B is a member of a subfamily of Rabs. A previous study revealed that vesicle exocytosis and the release of important growth regulators are controlled by $\mathrm{Rab} 27 \mathrm{~B}$, leading to the regulation of invasive growth and metastasis in human breast tumors (11). As OC and breast cancer possess a number of commonalities in terms of genetic mutations, the present study hypothesized whether the expression of Rab27B was correlated with the progression of OC.

In the current study, it was hypothesized that the expression of Rab27B was associated with the development and 
progression of $\mathrm{OC}$. In order to investigate the association between the expression of Rab27B and OC, and whether the expression level correlated with the 5-year survival rate, 204 samples were selected, which included 103 malignant tumors, 57 benign ovarian adenoid tumors and 44 borderline tumors. In addition, the correlation between staining intensity of Rab27B and clinical parameters was detected in order to assess the prognostic value of Rab27B in OC. The benign and borderline ovarian tumors served as control groups.

\section{Materials and methods}

Patients and tissue samples. A total of 204 samples, including 57 benign ovarian adenoid tumors, 44 borderline tumors and 103 malignant tumors, were acquired from patients who had undergone ovarian resection without preoperative systemic chemotherapy at the Surgical Department of the Affiliated Hospital of Nantong University (Nantong, China) between May 2004 and April 2013. The present study was approved by the Ethics Committee of the Affiliated Hospital of Nantong University (Nantong, China), and preoperative informed consent was obtained from each patient.

Fresh samples were processed immediately following surgical removal. The malignant tumors functioned as the experimental group, while the benign adenoid tumors and borderline tumors served as controls. Following attainment of informed consent, patients were interviewed to obtain information on demographic characteristics, and clinical data were collected. The patients were followed-up every 3 months for the first year, every 6 months for the subsequent 2 years and annually thereafter. If the patients were available for the full period, the follow-up time was 9 years, with a range of frequency from 2-107 months. Tumor grade was classified as low or high according to the MD Anderson grading criteria (12). Surgical staging was determined based on the International Federation of Obstetrics and Gynecology (FIGO) criteria. Staging results were as follows: Stage I, 49; stage II, 6; stage III, 48; and stage IV, 0 . Tumor grades were classified as high $(n=83)$ or low $(n=20)$. The primary clinical and pathological variables are shown in Table I.

Tissue microarray (TMA) construction and immunohistochemical (IHC) analysis. All tumor samples were embedded in paraffin (Qidong City Chemical Co., Ltd., Nantong, China) following fixation with $10 \%$ formaldehyde (Qidong City Chemical Co., Ltd.) for $24 \mathrm{~h}$, and used for constructing the TMA. A Tissue Microarray System (UT06 Quick-Ray ${ }^{\circledR}$; UNITMA, Seoul, Korea) was used. Core tissue biopsies (2 mm in diameter) were selected from individual paraffin-embedded sections and arranged in recipient paraffin blocks as described previously (13-15). IHC analysis was performed to detect the expression of Rab27B protein in samples. Paraffin tissue sections $(4 \mu \mathrm{m})$ were deparaffinized with $100 \%$ xylene and rehydrated using a graded alcohol series, followed by quenching in $3 \%$ hydrogen peroxide in order to block endogenous peroxidase activity. The sections were subsequently processed in $10 \mathrm{mM}$ citrate buffer ( $\mathrm{pH} \mathrm{6.0)}$ ) and heated to $121^{\circ} \mathrm{C}$ in an autoclave for $20 \mathrm{~min}$ for antigen retrieval. Following rinsing with phosphate-buffered saline (PBS; $\mathrm{pH} 7.2$ ), the sections were incubated at room temperature with rabbit anti-human Rab27B polyclonal antibody (catalog no., ab104472; 1:300 dilution; Abcam, Cambridge, MA, USA) for $2 \mathrm{~h}$. All slides were processed using the peroxidase-anti-peroxidase method, which consists of combining the primary antibody and the target antigen, and combining the horseradish peroxidase goat antirabbit IgG polyclonal secondary antibody (catalog no., SN135; 1:1,000; Beyotime Institute of Biotechnology, Haimen, China) and the peroxidase-anti-peroxidase (Beyotime Institute of Biotechnology) to form a complex. Following rinsing with PBS, the peroxidase reaction was visualized by incubating the sections in $0.1 \%$ phosphate buffer solution, $0.02 \%$ diaminobenzidine tetrahydrochloride (Shanghai Shifeng Biological Technology Co., Ltd., Shanghai, China) and 3\% hydrogen peroxide (Shanghai Shifeng Biological Technology Co., Ltd.). Following rinsing in water, the sections were counterstained using hematoxylin, dehydrated and coverslipped.

IHC evaluation. All immunostained sections were evaluated in a blinded manner, with no knowledge of the clinical and pathological parameters of the patients. Rab27B-positive cells were counted by monitoring at least 1,000 cells from at least five randomly selected fields. In order to allow statistical analyses, patients were divided into three groups according to the expression rate as follows: High expression group (100-75\%, score of 3), moderate expression group (50-75\%, score of 2) and low expression group ( $<50 \%$, score of 1$)$. For density evaluation, low staining was scored as 1 , moderate staining as 2 and intense staining as 3. The two scores were multiplied and samples were classified into two groups as follows: Score of 1 (0-4.5) and score of 2 (4.5-9; Table I). In half of the samples, staining was repeated three times to avoid potential technical errors and similar results were obtained. The evaluation procedures were performed by three independent pathologists using a Multi-Head microscope (Precise Instrument Co., Ltd., Beijing, China), and a consensus was achieved.

Statistical analysis. All computations were performed with SPSS version 17.0 (SPSS, Inc., Chicago, IL, USA). All values were expressed as the mean \pm standard error. Rab27B expression and clinicopathological features were analyzed with Pearson's $\chi^{2}$ test. Kaplan-Meier analysis and the log-rank test were used to analyze survival data. Univariate and multivariate analysis was performed using the Cox proportional hazards model, and the hazard ratio and $95 \%$ confidence interval were recorded for every marker. $\mathrm{P}<0.05$ was considered to indicate a statistically significant difference. Each experiment consisted of at least three replicates per condition.

\section{Results}

Rab27B expression levels vary in ovary epithelia from $O C$, borderline ovarian tumors and benign ovarian adenoid tumors. In order to investigate the protein expression profile of Rab27B in OC, IHC was initially performed in 103 OC samples, 57 benign ovarian adenoid tumors and 44 borderline tumor samples. Positive immunostaining was primarily observed in carcinoma cells in the cytoplasm and the nucleus. According to the results of the IHC experiments, all of the OC samples were positive for Rab27B, while the expression levels were relatively lower in borderline tumors. Furthermore, Rab27B expression was barely detectable in benign adenoid tumors (Fig. 1). 
A

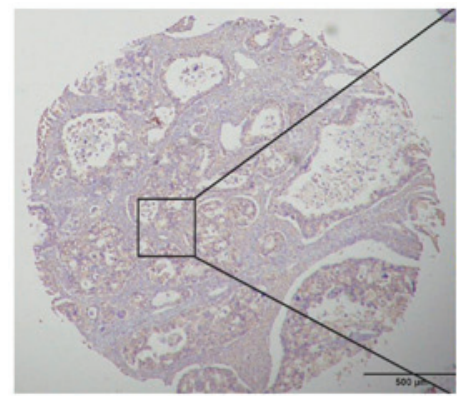

C

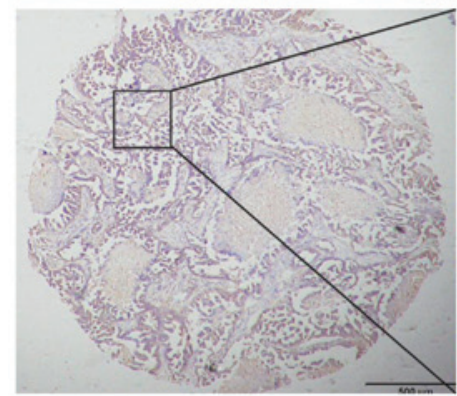

$\mathbf{E}$

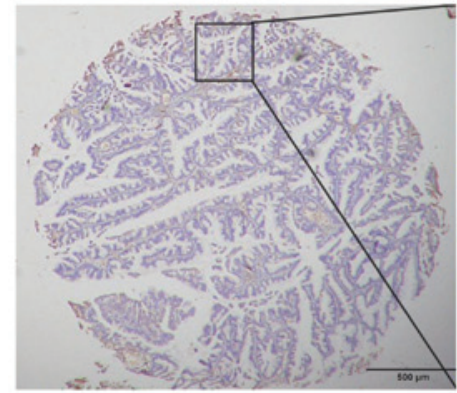

G

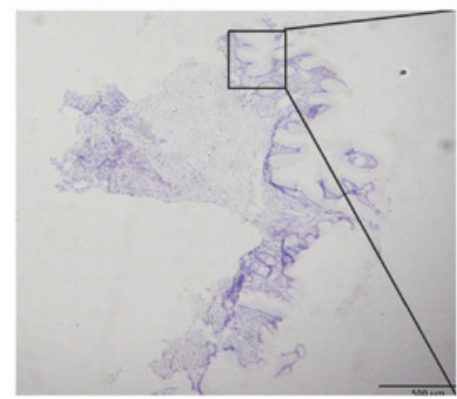

B

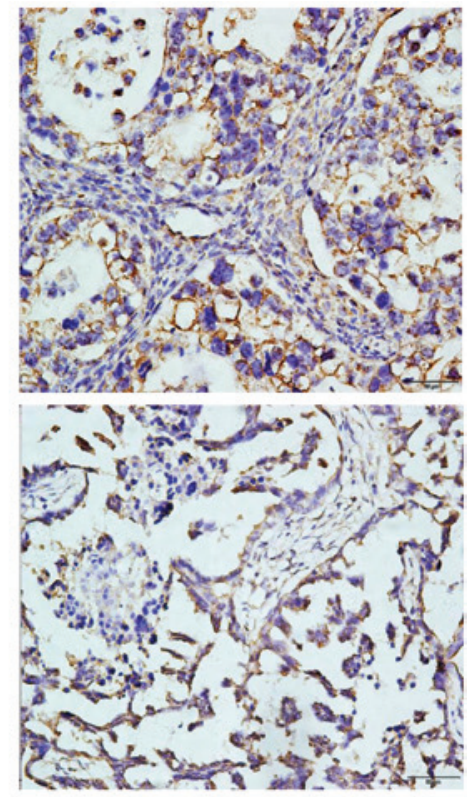

F

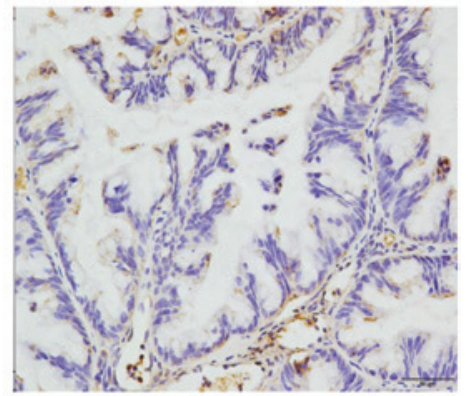

H

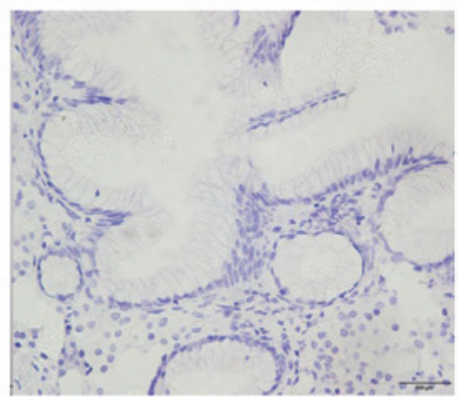

Figure 1. Immunohistochemically stained samples of malignant tumors, borderline tumors and benign adenoid tumors. (A-D) Positive staining of Rab27B in malignant tumors. (E and F) Weak staining of Rab27B in borderline tumors. ( $\mathrm{G}$ and $\mathrm{H}$ ) Negative staining of Rab27B in benign adenoid tumors. (A, C, E and G), magnification x40, (B, D, F and H), magnification x400.

Rab27B expression is associated with certain OC clinicopathological features. In order to investigate the physiological and pathological associations between the expression of Rab27B in $\mathrm{OC}$, the presence of Rab27B in various cancer grades and histological types is summarized in Table I. Rab27B was expressed at an increased level in poorly-differentiated specimens compared with well-differentiated specimens. It was identified that Rab27B expression was significantly associated with histological type $(\mathrm{P}=0.012)$, lymph node metastasis $(\mathrm{P}=0.024)$, distant metastasis $(\mathrm{P}<0.001)$, tumor grade $(\mathrm{P}=0.015)$ and tumor stage $(\mathrm{P}=0.001)$. However, there was no correlation between Rab27B expression and other prognostic factors, including age, unilateral or bilateral disease and ascites.

Elevated Rab27B expression predicts poor prognosis of $O C$. Univariate and multivariate analyses were performed in order to identify relevant prognostic factors for overall survival using the Cox proportional hazards model. Univariate analysis revealed that the following variables were significantly associated with a poorer prognosis: Rab27B expression $(\mathrm{P}<0.001)$, ascites $(\mathrm{P}=0.033)$, FIGO stage $(\mathrm{P}=0.001)$ and distant metastasis $(\mathrm{P}<0.001)$. In order to verify the accuracy of the results, multivariate analysis was performed by including significant $(\mathrm{P}<0.05)$ variables from the univariate analysis. However, the multivariate analysis revealed that only Rab27B expression $(\mathrm{P}<0.001)$ and distant metastasis $(\mathrm{P}=0.031)$ had independent prognostic effects on the overall survival of patients with $\mathrm{OC}(\mathrm{P}<0.0001$; Table II). Kaplan-Meier survival curves revealed that OC patients with low or no Rab27B expression, and patients without distant metastasis, possessed a more favorable prognosis (Fig. 2A and $\mathrm{B}$ ). 
Table I. Associations between Rab27B cytoplasm expression and clinicopathological features.

\begin{tabular}{|c|c|c|c|c|c|}
\hline \multirow[b]{2}{*}{ Clinicopathological feature } & \multirow[b]{2}{*}{$\mathrm{n}$} & \multicolumn{2}{|c|}{ Rab27B expression } & \multirow[b]{2}{*}{$\chi^{2}$} & \multirow[b]{2}{*}{ P-value } \\
\hline & & Low & High & & \\
\hline Histological type & & & & 10.972 & $0.012^{\mathrm{a}}$ \\
\hline Serous carcinoma & 79 & 33 & 46 & & \\
\hline Adenocarcinoma & 11 & 6 & 5 & & \\
\hline Endometrioid & 4 & 0 & 4 & & \\
\hline Other & 9 & 8 & 1 & & \\
\hline Age (years) & & & & 2.979 & 0.084 \\
\hline$<60$ & 61 & 33 & 28 & & \\
\hline$\geq 60$ & 42 & 14 & 28 & & \\
\hline Unilateral or bilateral & & & & 3.166 & 0.075 \\
\hline Unilateral & 65 & 34 & 31 & & \\
\hline Bilateral & 38 & 13 & 25 & & \\
\hline Ascites & & & & 0.941 & 0.332 \\
\hline No & 53 & 26 & 27 & & \\
\hline Yes & 41 & 16 & 25 & & \\
\hline Unknown & 9 & 5 & 4 & & \\
\hline Tumor grade & & & & 5.941 & $0.015^{\mathrm{a}}$ \\
\hline Low & 20 & 14 & 6 & & \\
\hline High & 83 & 33 & 50 & & \\
\hline Lymph node metastasis & & & & 4.619 & $0.024^{\mathrm{a}}$ \\
\hline No metastasis & 82 & 42 & 40 & & \\
\hline Metastasis & 21 & 5 & 16 & & \\
\hline Distant metastasis & & & & 20.246 & $<0.001^{\mathrm{a}}$ \\
\hline No metastasis & 54 & 36 & 18 & & \\
\hline Metastasis & 49 & 11 & 38 & & \\
\hline Tumor stage & & & & 14.667 & $0.001^{\mathrm{a}}$ \\
\hline I & 49 & 32 & 17 & & \\
\hline II & 6 & 2 & 4 & & \\
\hline III & 48 & 13 & 35 & & \\
\hline
\end{tabular}

Statistical analyses were performed by Pearson's $\chi^{2}$ test. ${ }^{\mathrm{a}} \mathrm{P}<0.05$ was considered to indicate a statistically significant difference.

A

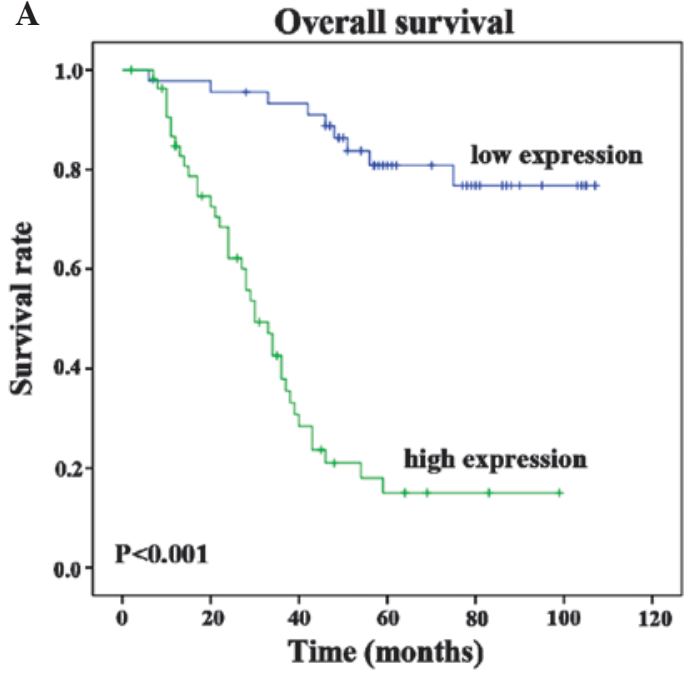

B

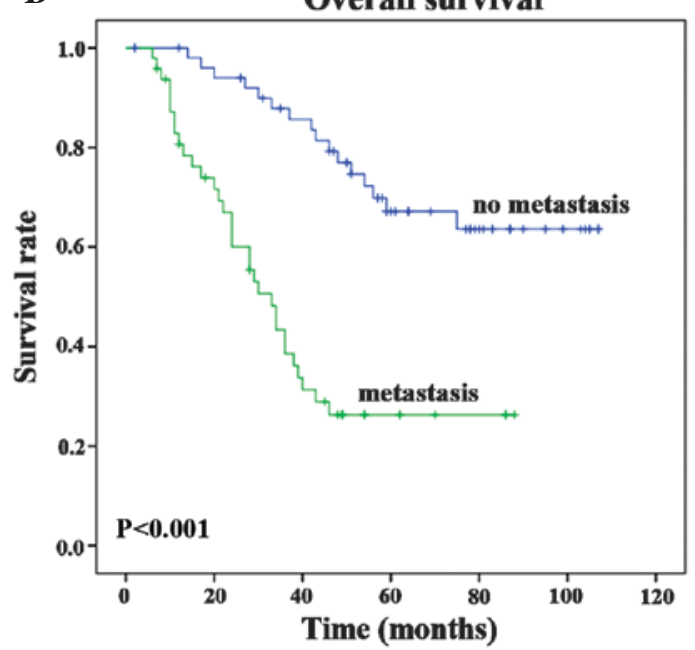

Figure 2. Kaplan-Meier survival curves revealed that OC patients with low or no Rab27B expression and patients without distant metastases possessed a more favorable prognosis. Correlation between patient survival rates and (A) the expression of Rab27B and (B) distant metastasis. 
Table II. Contribution of various potential prognostic factors to survival investigated by Cox regression analysis in 103 ovarian cancer specimens.

A, Univariate analysis

\begin{tabular}{|c|c|c|c|c|c|}
\hline Variable & Type & Years & Hazard ratio & 95\% Confidence interval & P-value \\
\hline Expression & High vs. low & 5 & 8.821 & $4.171-18.656$ & $<0.001^{\mathrm{a}}$ \\
\hline Age & $<60$ vs. $\geq 60$ & 5 & 1.474 & $0.832-2.261$ & 0.183 \\
\hline Histology type & Serous vs. others & 5 & 0.912 & $0.479-1.771$ & 0.805 \\
\hline Ascites & Yes vs. no & 5 & 1.894 & $1.052-3.480$ & $0.033^{\mathrm{a}}$ \\
\hline Differentiation & High grade vs. low grade & 5 & 1.904 & $0.808-4.482$ & 0.141 \\
\hline FIGO stage & FIGO I-II vs. FIGO III & 5 & 2.755 & $1.544-4.987$ & $0.001^{\mathrm{a}}$ \\
\hline Lymph nodes metastasis & No metastasis vs. metastasis & 5 & 1.543 & $0.801-2.972$ & 0.195 \\
\hline Unilateral or bilateral & Unilateral vs. bilateral & 5 & 0.025 & $1.083-3.375$ & 1.912 \\
\hline Distant metastasis & Metastasis vs. no metastasis & 5 & 4.188 & 2.264-7.748 & $<0.001^{\mathrm{a}}$ \\
\hline
\end{tabular}

B, Multivariate analysis

\begin{tabular}{|c|c|c|c|c|c|}
\hline Variable & Type & Years & Hazard ratio & $95 \%$ Confidence interval & P-value \\
\hline Expression & High vs. low & 5 & 8.471 & $3.526-20.350$ & $<0.001^{\mathrm{a}}$ \\
\hline Age & $<60$ vs. $\geq 60$ & 5 & 0.709 & $0.364-1.383$ & 0.313 \\
\hline Histology type & Serous vs. others & 5 & 1.839 & $0.893-3.790$ & 0.098 \\
\hline Ascites & Yes vs. no & 5 & 1.44 & $0.716-2.895$ & 0.306 \\
\hline Differentiation & High grade vs. low grade & 5 & 1.132 & $0.394-3.252$ & 0.818 \\
\hline FIGO stage & FIGO I-II vs. FIGO III & 5 & 1.269 & $0.535-3.009$ & 0.589 \\
\hline Lymph nodes metastasis & No metastasis vs. metastasis & 5 & 0.961 & $0.479-1.925$ & 0.910 \\
\hline Unilateral or bilateral & Unilateral vs. bilateral & 5 & 0.874 & $0.437-1.747$ & 0.702 \\
\hline Distant metastasis & Metastasis vs. no metastasis & 5 & 2.622 & $1.091-6.302$ & $0.031^{\mathrm{a}}$ \\
\hline
\end{tabular}

${ }^{\mathrm{a}} \mathrm{P}<0.05$. FIGO; International Federation of Gynecology and Obstetrics.

\section{Discussion}

Rab proteins are regulators of vesicular transport, which interconnect membrane-bound organelles and have been hypothesized to have significant roles in the targeting, docking and fusion of vesicular carriers due to their specific acceptor compartments (16). The majority of Rab proteins perform identical basic functions in all cells, whereas others are only observed in certain cell types, where they perform specialized functions. The Rab27 subfamily members, which include the homologues Rab27A and B, are 71\% identical at the amino acid level and have been detected in several secretory tissues and hematopoietic cells (17-19). Rab27B may enhance proliferation under limiting serum concentrations, via increased G1-S-phase cell cycle transition. This functional response is Rab27B specific, and is dependent upon GTP binding and membrane anchorage (16). In the present study, in order to investigate the expression of Rab27B in ovary tissues, the immunoreactivity of Rab27B was evaluated in malignant ovarian tumors, borderline ovarian tumors and benign ovarian adenoid tumors. The results of the present study demonstrated that all of the OC samples were positive for Rab27B expression, while expression levels were relatively reduced in borderline tumors. Furthermore, Rab27B expression was barely detectable in benign adenoid tumors. Thus, it was concluded that the expression levels of Rab27B increase in ovary tissues with the development of ovary tumors, and Rab27B expression levels may increase further with the occurrence of cancer.

A previous study revealed that Rab family members are closely associated with the progression of tumors (20). It has been demonstrated that Rab coupling protein contributes to the malignant progression of squamous cell carcinoma of the head and neck (SCCHN), and serves as a novel prognostic marker in patients with SCCHN (20). Rab25 is able to inhibit apoptosis, as well as enhance the proliferation and invasion of tumor cells. Furthermore, Rab25 additionally demonstrated an association with the progression of breast cancer and OC (21-23). Knockdown of Rab25 may reduce cell cycle progression and induce apoptosis in OC cells (24). A previous study additionally demonstrated that Rab35 was expressed at a high level in OC (25). Another previous study performed a detailed analysis in order to elucidate the correlation between Rab27B expression and clinicopathological variables of OC (26). It was revealed that Rab27B expression was closely associated with the progression and tumor stage of hepatocellular carcinoma; furthermore, it was additionally demonstrated that patients with Rab27B positivity possessed significantly reduced overall survival (26). According to the 
results of the present study, Rab27B was expressed at a higher level in poorly-differentiated specimens compared with well-differentiated specimens. The expression of Rab27B was additionally associated with histological type, tumor grade and tumor stage. Thus, the present study suggested that Rab27B may be a significant factor for identification of the severity of $\mathrm{OC}$.

It is known that tumor invasion and metastasis is the cause of the majority of cancer-associated mortalities. A previous study revealed that Rab27B was upregulated in breast cancer, and this upregulation coincided with lymph node metastasis and acquisition of a poor prognostic phenotype (27). An in vitro experiment demonstrated that breast cancer cells with Rab27B overexpression formed cellular extensions and a spreading morphology, and possessed a significantly increased ability to invade Matrigel and native type I collagen substrates (28). This invasion was evidenced by increased tumor volume, tumor weight and Ki67 proliferation index in xenograft models (28). In the present study, it was identified that patients with increased Rab27B expression were more likely to have lymph node and distant metastases. This finding may assist with the identification of patients who require chemotherapy or radiotherapy. In light of the results of the present study, it was hypothesized that Rab27B may be a novel predictor of metastasis in OC patients.

Clinically elevated levels of Rab27B expression were observed to be associated with poor prognosis in patients with bladder cancer (29). Furthermore, Rab27B has additionally been identified as a predictor of prognosis in gastrointestinal stromal tumors (30). In the present study, the mean 5-year survival rate of all patients with OC $(n=52)$ was $50 \%$. When the samples were divided into two groups according to the expression of Rab27B [score of 1 (0-4.5) and score of 2 (4.5-9] the survival rate decreased along with the staining intensity. The Kaplan-Meier curve revealed a cumulative 5-year survival rate of $80 \%$ for patients with low expression of Rab27B compared with $29.1 \%$ for patients with high expression of Rab27B. The results of the present study indicated that Rab27B-positive tumors were significantly correlated with poor overall survival. Multivariate analysis revealed that OC patients with increased Rab27B expression and distant metastases possessed more negative survival outcomes compared with those expressing lower levels of Rab27B. The results of the present study were consistent with previous findings demonstrating that upregulated Rab27B expression is a poor prognostic factor, suggesting the clinical value of Rab27B for assessment of the prognosis of OC patients, meaning that Rab27B may be a novel and significant prognostic marker for OC patients.

$\mathrm{OC}$ is a common gynecological malignancy and a significant cause of cancer-associated mortality among women (31). Although $6 \%$ of all cancer-associated mortalities in women are caused by OC, survival rates have not improved significantly over the past decade (32). Despite advances in therapeutic methods, the prognosis of patients with OC remains poor, particularly for those with FIGO stage III and IV disease. The recurrence time is frequently $<2$ years. The 5-year survival rate for FIGO stage III disease is $20-25 \%(33,34)$. Therefore, the identification of factors associated with prognosis and clinical stage would be of value for the development of novel therapies and improvement of survival rates.

To the best of our knowledge, the current study is the first to examine Rab27B expression in OC. The present study identified that Rab27B was upregulated in OC, and Rab27B overexpression was significantly correlated with tumor progression and poor survival rate. This indicated that Rab27B may serve as a specific and novel prognostic marker in OC. However, additional studies are required in order to determine the underlying molecular mechanism of Rab27B involvement in OC progression and prognosis. This may lead to future development of novel approaches to the treatment of OC by targeting Rab27B, allowing effective tumor management.

\section{Acknowledgements}

The present study was supported by Postdoctoral study grants (grant no. 2013-40-5) and Translational Medicine Research (grant no. TDFzh2014011) from the Affiliated Hospital of Nantong University (Nantong, China).

\section{References}

1. Goker A, Guvenal T, Yanikkerem E, Turhan A and Koyuncu FM: Quality of life in women with gynecologic cancer in Turkey. Asian Pac J Cancer Prev 12: 3121-3128, 2011.

2. Zhang B, Cai FF and Zhong XY: An overview of biomarkers for the ovarian cancer diagnosis. Eur J Obstet Gynecol Reprod Biol 158: 119-123, 2011.

3. Jemal A, Siegel R, Ward E, Murray T, Xu J and Thun MJ: Cancer statistics, 2007. CA Cancer J Clin 57: 43-66, 2007.

4. Matsuda A and Katanoda K: Five-year relative survival rate of ovarian cancer in the USA, Europe and Japan. Jpn J Clin Oncol 44: 196, 2014

5. Fukuda M: Regulation of secretory vesicle traffic by Rab small GTPases. Cell Mol Life Sci 65: 2801-2813, 2008.

6. Grosshans BL, Ortiz D and Novick P: Rabs and their effectors: Achieving specificity in membrane traffic. Proc Natl Acad Sci USA 103: 11821-11827, 2006.

7. Pfeffer SR: Structural clues to Rab GTPase functional diversity. J Biol Chem 280: 15485-15488, 2005.

8. Paul P, Simm S, Mirus O, Scharf KD, Fragkostefanakis S and Schleiff E: The complexity of vesicle transport factors in plants examined by orthology search. PLoS ONE 9: e97745, 2014.

9. Pfeffer S: A model for Rab GTPase localization. Biochem Soc Trans 33: 627-630, 2005.

10. Wright PK: Targeting vesicle trafficking: An important approach to cancer chemotherapy. Recent Pat Anticancer Drug Discov 3: 137-147, 2008.

11. Hendrix A, Braems G, Bracke M, Seabra M, Gahl W, De Wever O and Westbroek W: The secretory small GTPase Rab27B as a marker for breast cancer progression. Oncotarget 1: 304-308, 2010.

12. Malpica A, Deavers MT, Lu K, Bodurka DC, Atkinson EN, Gershenson DM and Silva EG: Grading ovarian serous carcinoma using a two-tier system. Am J Surg Pathol 28: 496-504, 2004.

13. Feng J, Xu L, Ni S, Gu J, Zhu H, Wang H, Zhang S, Zhang W and Huang J: Involvement of FoxQ1 in NSCLC through regulating EMT and increasing chemosensitivity. Oncotarget 5: 9689-9702, 2014.

14. Huang J, Zhang J, Li H, Lu Z, Shan W, Mercado-Uribe I and Liu J: VCAM1 expression correlated with tumorigenesis and poor prognosis in high grade serous ovarian cancer. Am J Transl Res 5: 336-346, 2013.

15. Feng J, Zhang X, Zhu H, Wang X, Ni S and Huang J: High expression of FoxP1 is associated with improved survival in patients with non-small cell lung cancer. Am J Clin Pathol 138: 230-235, 2012.

16. Dong W, Mou Q, Chen J, Cui J, Li W, and Xiao W: Differential expression of Rab27A/B correlates with clinical outcome in hepatocellular carcinoma. World J Gastroenterol 18:1806-1813, 2012. 
17. Westbroek W, Tuchman M, Tinloy B, De Wever O, Vilboux T, Hertz JM, Hasle H, Heilmann C, Helip-Wooley A, Kleta R, and Gahl WA: A novel missense mutation (G43S) in the switch I region of Rab27A causing Griscelli Syndrome. Mol Genet Metab 94: 248-254, 2008.

18. Westbroek W, Lambert J, De Schepper S, Kleta R, Van Den Bossche K, Seabra MC, Huizing M, Mommaas M and Naeyaert JM: Rab27b is up-regulated in human Griscelli syndrome type II melanocytes and linked to the actin cytoskeleton via exon F-myosin Va transcripts. Pigment Cell Res 17: 498-505, 2004

19. Jordens I, Westbroek W, Marsman M, Rocha N, Mommaas M, Huizing M, Lambert J, Naeyaert JM and Neefjes J: Rab7 and Rab27a control two motor protein activities involved in melanosomal transport. Pigment Cell Res 19: 412-423, 2006.

20. Dai Y, Liu Y, Huang D, Yu C, Cai G, Pi L, Ren C, Chen GZ, Tian Y and Zhang X: Increased expression of Rab coupling protein in squamous cell carcinoma of the head and neck and its clinical significance. Oncol Lett 3: 1231-1236, 2012.

21. Cheng KW, Lahad JP, Kuo WL, Lapuk A, Yamada K, Auersperg N, Liu J, Smith-McCune K, Lu KH, Fishman D, et al: The RAB25 small GTPase determines aggressiveness of ovarian and breast cancers. Nat Med 10: 1251-1256, 2004.

22. Cheng KW, Lahad JP, Gray JW and Mills GB: Emerging role of RAB GTPases in cancer and human disease. Cancer Res 65: 2516-2519, 2005.

23. Fan Y, Xin XY, Chen BL and Ma X: Knockdown of RAB25 expression by RNAi inhibits growth of human epithelial ovarian cancer cells in vitro and in vivo. Pathology 38: 561-567, 2006.

24. Liu Y, Tao X, Jia L, Cheng KW, Lu Y, Yu Y and Feng Y: Knockdown of RAB25 promotes autophagy and inhibits cell growth in ovarian cancer cells. Mol Med Rep 6: 1006-1012, 2012

25. Subramani D and Alahari SK: Integrin-mediated function of Rab GTPases in cancer progression. Mol Cancer 9: 312, 2010 .
26. Dong WW, Mou Q, Chen J, Cui JT, Li WM and Xiao WH Differential expression of Rab27A/B correlates with clinical outcome in hepatocellular carcinoma. World J Gastroenterol 18: 1806-1813, 2012

27. Zhang JX, Huang XX, Cai MB, Tong ZT, Chen JW, Qian D, Liao YJ, Deng HX, Liao DZ, Huang MY, et al: Overexpression of the secretory small GTPase Rab27B in human breast cancer correlates closely with lymph node metastasis and predicts poor prognosis. J Transl Med 10: 242, 2012.

28. Hendrix A, Westbroek W, Bracke M and De Wever O: An ex(o)citing machinery for invasive tumor growth. Cancer Res 70: 9533-9537, 2010.

29. Ostenfeld MS, Jeppesen DK, Laurberg JR, Boysen AT, Bramsen JB, Primdal-Bengtson B, Hendrix A, Lamy P, Dagnaes-Hansen F, Rasmussen MH, et al: Cellular disposal of miR23b by RAB27-dependent exosome release is linked to acquisition of metastatic properties. Cancer Res 74: 5758-5771, 2014.

30. Wang W, Ni Q, Wang H, Zhang S and Zhu H: Prognostic value of Rab27B nuclear expression in gastrointestinal stromal tumors. Dis Markers 2014: 942181, 2014.

31. Xu YZ, Xi QH, Ge WL and Zhang XQ: Identification of serum microRNA-21 as a biomarker for early detection and prognosis in human epithelial ovarian cancer. Asian Pac J Cancer Prev 14: 1057-1060, 2013.

32. Siegel R, Naishadham D and Jemal A: Cancer statistics, 2012. CA Cancer J Clin 62: 10-29, 2012.

33. Lambert HE, Gregory WM, Nelstrop AE and Rustin GJ: Long-term survival in 463 women treated with platinum analogs for advanced epithelial carcinoma of the ovary: Life expectancy compared to women of an age-matched normal population. Int J Gynecol Cancer 14: 772-778, 2004.

34. McGuire WP 3rd: Current status of taxane and platinum-based chemotherapy in ovarian cancer. J Clin Oncol 21: S133-S135, 2003 . 